\title{
RESEARCH
}

Open Access

\section{Pilot study of a basic individualized cognitive behavioral therapy program for chronic pain in Japan}

Hiroki Hosogoshi ${ }^{1,2 *}$, Kazunori Iwasa ${ }^{3}$, Takaki Fukumori ${ }^{4}$, Yuriko Takagishi ${ }^{2,5}$, Yoshitake Takebayashi ${ }^{2,6}$, Tomonori Adachi ${ }^{7}$, Yuki Oe ${ }^{2,8}$, Yukino Tairako ${ }^{2,9}$, Yumiko Takao ${ }^{10}$, Hiroyuki Nishie ${ }^{11}$, Ayako Kanie ${ }^{2}$, Masaki Kitahara ${ }^{12}$, Kiyoka Enomoto ${ }^{13}$, Hirono Ishii ${ }^{14}$, Issei Shinmei ${ }^{15,16}$, Masaru Horikoshi ${ }^{2}$ and Masahiko Shibata ${ }^{17}$

\begin{abstract}
Background: Chronic pain is a major health problem, and cognitive behavioral therapy (CBT) is its recommended treatment; however, efforts to develop CBT programs for chronic pain and assess their feasibility are remarkably delayed in Asia. Therefore, we conducted this pilot study to develop a basic individualized CBT for chronic pain (CBT-CP) and assessed its feasibility for use in Japan.
\end{abstract}

Methods: Our study was an open-labeled before-after trial without a control group conducted cooperatively in five Japanese tertiary care hospitals. Of 24 outpatients, 15, age 20-80, who experienced chronic pain for at least three months were eligible. They underwent an eight-session CBT-CP consisting of relaxation via a breathing method and progressive muscle relaxation, behavioral modification via activity pacing, and cognitive modification via cognitive reconstruction. The EuroQol five-dimensional questionnaire five level (EQ5D-5 L) assessment as the primary outcome and quality of life (QOL), pain severity, disability, catastrophizing, self-efficacy, and depressive symptoms as secondary outcomes were measured using self-administered questionnaires at baseline, posttreatment, and 3-month follow-up. Intention-to-treat analyses were conducted.

Results: Effect size for EQ5D-5 L score was medium from baseline to post-treatment (Hedge's $g=-0.72,90 \%$ confidence interval $=-1.38$ to -0.05$)$ and up to the 3-month follow-up ( $g=-0.60, \mathrm{Cl}=-1.22$ to 0.02$)$. Effect sizes for mental and role/social QOL, disability, catastrophizing, self-efficacy, and depressive symptoms were medium to large, although those for pain severity and physical QOL were small. The dropout rate was acceptably low at $14 \%$. No severe adverse events occurred.

Conclusion: The findings suggest that CBT-CP warrants a randomized controlled trial in Japan.

(Continued on next page)

\footnotetext{
* Correspondence: hosogosh@kansai-u.ac.jp

${ }^{1}$ Department of Sociology, Faculty of Sociology, Kansai University, 3-3-35

Yamate-cho, Suita-shi, Osaka 564-8680, Japan

${ }^{2}$ National Center for Cognitive Behavior Therapy and Research, National

Center of Neurology and Psychiatry, 4-1-1 Ogawahigashi-cho, Kodaira-shi,

Tokyo 187-8551, Japan

Full list of author information is available at the end of the article
}

\section{$\triangle B M C$}

(c) The Author(s). 2020 Open Access This article is licensed under a Creative Commons Attribution 4.0 International License, which permits use, sharing, adaptation, distribution and reproduction in any medium or format, as long as you give appropriate credit to the original author(s) and the source, provide a link to the Creative Commons licence, and indicate if changes were made. The images or other third party material in this article are included in the article's Creative Commons licence, unless indicated otherwise in a credit line to the material. If material is not included in the article's Creative Commons licence and your intended use is not permitted by statutory regulation or exceeds the permitted use, you will need to obtain permission directly from the copyright holder. To view a copy of this licence, visit http://creativecommons.org/licenses/by/4.0/ The Creative Commons Public Domain Dedication waiver (http://creativecommons.org/publicdomain/zero/1.0/) applies to the data made available in this article, unless otherwise stated in a credit line to the data. 
(Continued from previous page)

Trial registration: University Hospital Medical Information Network Clinical Trials Registry (UMIN-CTR), UMIN00002 0880. Registered on 04 February 2016.

Keywords: Chronic pain, Cognitive behavioral therapy, Psychotherapy, Multidisciplinary treatment, Quality of life (QOL), Japanese, Asia, Feasibility,

\section{Background}

Chronic pain is pain that persists past normal healing time and lasts for $>3-6$ months $[1,2]$. It is related to disability, catastrophizing, and mood disorders such as depression. These conditions affect not only the patients but also their family, workplace, and society and negatively affect their quality of life (QOL) [2-4]. The prevalence of chronic pain is as high as $20 \%$ [5] and is rising every year [6]. The annual economic costs of pain associated with lower worker productivity range from $\$ 560$ to $\$ 635$ billion in the US [7].

Multidisciplinary chronic pain management based on bio-psycho-social models have been recommended, and cognitive behavioral therapy (CBT) is considered especially important in the treatment of chronic pain [8]. CBT addresses various problems caused by chronic pain rather than the pain per se $[9,10]$; these interventions include decreasing maladaptive pain behavior and increasing adaptive behavior, identifying and modifying irrational beliefs, heightening self-efficacy to pain management, reducing psychological stress, improving physical and social function, and raising QOL. The efficacy of CBT for chronic pain (CBT-CP) has been established by many randomized controlled trials (RCT) outside Japan [11-14]. According to a systematic review [15], the effect of CBT on pain per se remains small and short term, but its effects on disability, catastrophizing, and mood are sustained over the medium and long terms. CBT-CP is also effective in improving QOL $[14,16]$.

Chronic pain is a serious problem in Japan as well. The prevalence of chronic pain is as high as $22.9 \%$ [17], and the annual economic costs of work loss due to chronic pain was estimated to be $\$ 19.9$ billion [3]. Although CBT is recommended in Japanese treatment guidelines [18-20], manual therapy and nerve block injection are the mainstream approaches [21] and studies addressing the effect of CBT-CP in Japan have been few and insufficient. Matsubara et al. [22] conducted a study examining the effect of individual CBT among Japanese chronic pain patients, wherein treatment responders were just compared with non-responders; moreover, according to Yang et al. [23], the technical quality of therapists in that study was not well secured. Another study was a before-after trial of group CBT [24]. Group CBT has potential utility for patients through the modeling of appropriate responses from others and decreasing the patient's feelings of being isolated and misunderstood [24], but the effect of CBT-CP itself has not yet been sufficiently verified in Japan; thus, it is a priority to establish a basic individualized CBT-CP program. This will promote the popularization of CBT-CP in Japanese clinical settings. In this context, the delay in $\mathrm{CBT}$ research and practice for chronic pain is a significant factor not only in Japan but also in all of Asia. It will be necessary to develop treatment methods and conduct welldesigned studies to verify the effect of CBT-CP in Asia, considering cultural differences [23].

We conducted this study to develop an individualized CBT-CP program for Japanese patients, to assess its feasibility, and to inform decisions regarding sample size for future definitive trials in terms of 1) treatment efficacy with QOL as the primary outcome and pain severity, disability, psychological variables, and depression as secondary outcomes; 2) acceptability (i.e., dropout rate); and 3) safety (i.e., severe adverse events). Treatment efficacy was assessed on the basis of changes in the primary and secondary outcome measures. We expected that statistically significant changes from baseline to posttreatment and up to follow-up would be found, that the dropout rate would be $<20 \%$, and that no severe adverse events would occur. A total of 40 studies that gave the number of participants at baseline and post-treatment assessment are referred to in a systematic review [15]. The mean dropout rate was $12.6 \%$ (standard deviation, 9.6; range, $0-34.7 \%$ ). Although most of the data is for anxiety disorders, not chronic pain, the dropout rates for general CBT in controlled trials are typically $5.6-19.0 \%$ [25]. Therefore, we set a dropout rate of $<20 \%$ in this study as adequate to show the feasibility of CBT-CP.

A previous study reported that CBT-CP is effective in reducing disability, especially among patients who are significantly disabled [26]. Therefore, we also explored the hypothesis that CBT-CP would be effective in increasing QOL among patients with low QOL.

\section{Methods}

\section{Trial design and ethics}

The study was a pilot, open-labeled, before-after trial without a control group. We conducted the CBT-CP program among Japanese chronic pain patients and evaluated health- 
related QOL at baseline, post-treatment, and 3-month follow-up. The study was conducted cooperatively in five tertiary care hospitals in Japan, and the participants were recruited from each hospital. The five sites were Osaka University Hospital, Kobe University Hospital, Kawasaki Medical School Hospital, National Center of Neurology and Psychiatry Hospital, and Jikei University Hospital; ethics committees at all sites evaluated the protocols (approval numbers 15429-2; 280046; 2275-2; A2016-092; and 28256 , respectively). The study was registered in the University Hospital Medical Information Network Clinical Trials Registry (UMIN-CTR: UMIN000020880).

\section{Participants, consent, and permissions}

Participants were outpatients who visited each site to receive medical treatment for their chronic pain. After complete explanations of the purpose and procedures of the study, we evaluated patient eligibility if they agreed to participate and signed the consent document. Patients who met the eligibility criteria were registered as participants and underwent baseline assessment. We conducted the entire eight-session CBT-CP program for all the participants. Participants underwent post-treatment assessment at $1 \pm 1$ week and a 3 -month follow-up assessment at $12 \pm 2$ weeks after the last session. No incentive was given for participation.

Inclusion criteria for the study were the following: a) Chronic pain persisting for at least 3 months, b) age 20-80 years, and c) understanding of the study and informed consent based on free will. Criterion a) was set for the examination of efficacy and b) and c) were set in consideration of informed consent. Exclusion criteria were the following: a) Organic causes of pain requiring immediate medical treatment, b) alcohol or substance use disorder, c) manic episodes or diagnosis of any psychotic disorder, d) severe suicidal ideation, e) difficulty in cognitive function required to undergo CBT, f) difficulty in communication or reading and writing in Japanese, g) chronic pain due to surgery or external injury, h) compensation or lawsuit related to the chronic pain, i) pain severity 10 in a numerical rating scale $(0-10)$, or $j)$ unsuitability due to any other reason as judged by the attending physician. Criteria a) $-\mathrm{f}$ ) and $\mathrm{j}$ ) were set in consideration of safety and excluding patients who would have difficulty receiving CBT-CP continuously and stably. Criteria g) and h) were set in consideration of a potential or actual financial gain from illness. Criterion i) was set as an expert opinion of our project team that patients with 10 points tend to adhere to seeking perfect elimination of their pain and were not fit for CBT-CP.

\section{Outcome measures}

EuroQol five-dimensional questionnaire five level (EQ5D-5 L) Health-related QOL was assessed using the EQ5D-5 L [27]. We used the Japanese version of EQ5D-5 L and a
Japanese scoring system that have been found to be valid and reliable [28]; a score of zero indicates death, and a score of one indicates complete health. The EQ5D-5 L score was the primary outcome.

\section{Medical outcomes in the 12-item short form health survey (SF-12)}

Mental and physical health-related QOL indicators were assessed using SF-12, which consists of a physical component summary (SF-12-PCS) and a mental component summary (SF-12-MCS) [29]. In Asia, including Japan, inclusion of a role/social component summary (SF-12RCS) is recommended, and its validity has been reported [30]. The summary scores are presented as T-scores with a mean of $50 \pm 10$ standard deviation, with a higher score indicating a healthier status.

\section{Numerical rating scale (NRS)}

Pain severity was assessed using NRS with anchors of 0 , indicating no pain, to 10 , indicating the worst pain [31]. Participants were asked to recall the most recent week and respond with a pain score indicating 1) maximum, 2) minimum, 3) average, and 4) current. The average value of these four scores was used for analysis because it is more useful when maximal reliability is necessary [31, 32]. A higher average score indicates more severe pain.

\section{Pain disability assessment scale (PDAS)}

To assess disability, PDAS was used [33, 34]. PDAS measures disability in terms of capability to do physical exercise and movement among chronic pain patients. Its reliability and validity has been reported [33, 34]. A higher score indicates a higher degree of disability due to pain.

\section{Patient health Questionnaire-9 (PHQ-9)}

PHQ-9 is a scale for assessing mental disorders common in primary care settings per the Diagnostic and Statistical Manual of Mental Disorders [35]. We used the Japanese version of PHQ-9, for which the reliability and validity have been reported [36]. A higher score on the scale indicates lower mental health.

\section{Pain Catastrophizing scale (PCS)}

We used PCS to assess cognitive factors that sustain chronic pain [37, 38]. The Japanese version of PCS has been found to be valid and reliable [39]; a higher score on the scale indicates a higher degree of catastrophizing about pain.

\section{Tampa scale for Kinesiophobia eleven (TSK-11)}

To assess fear of movement due to musculoskeletal pain, the Japanese version of TSK-11, which has sufficient validity and reliability, was used [40]; a higher score indicates a greater fear of movement. 
Pain self-efficacy questionnaire (PSEQ)

To assess the degree of confidence in performing a number of activities despite pain, we used PSEQ [41]. The Japanese version has been found to be valid and reliable [42]; a higher score indicates higher self-efficacy to cope with pain.

\section{Sample size}

We based our sample size on the recommendation that $\geq 12$ participants are suitable for pilot studies with a primary focus of estimating average values and variability to plan larger subsequent studies [43], because it was difficult to estimate sample size based on actual data due to the lack of sufficient data regarding CBT for Japanese chronic pain patients. Based on the above recommendation, 15 participants were targeted for recruitment in this study, considering dropouts.

\section{Interventions and clinical psychologists}

The CBT-CP developed for this study is a structured and manualized eight-session program. We prepared a workbook and a worksheet with several entry columns, including one for participants to write their own examples or experiences. Each session lasted approximately 30-40 min. The program comprised three components specifically addressing chronic pain; we conducted a narrative review of eight clinical trials reported during 2009-2014 [44-51] and found that behavioral modification, cognitive modification, and relaxation were common to these trials. The outline of each session is presented in Table 1; education and information regarding the CBT model and goal setting during session 1, relaxation via a breathing method and progressive muscle relaxation during session 2 , behavioral modification via activity pacing during sessions $3-5$, cognitive modification via cognitive reconstruction during sessions 6-7, and summary and relapse prevention during session 8 .

We did not use the third-generation CBT program (e.g., mindfulness-based stress reduction and acceptance and commitment therapy), because the effect sizes in the second- and third-generation CBT programs were almost equivalent at the time we developed our CBT-CP program. In addition, we surmised that the secondgeneration CBT would be more prevalent in Japan because there is more Japanese educational content regarding the second CBT than the third CBT program. The treatment sessions were conducted by six clinical psychologists (two with an M.A. and four with a Ph.D.) who fulfilled the following conditions: 1 ) $\geq 2$ years of practice experience dealing with CBT or experience of medical examination of chronic pain patients and 2) at least one patient's therapy fully supervised by $\mathrm{MH}$ and receiving approval as a CBT-CP practitioner. $\mathrm{MH}$ has been an expert in CBT for $>20$ years. Treatment adherence was
Table 1 Overview of our CBT-CP program

\begin{tabular}{ll}
\hline Session & Components \\
\hline 1 & Education and information regarding \\
& CBT (CBT model, self-monitoring) and goal \\
& setting \\
& Relaxation training (breathing method, \\
& progressive muscle relaxation) \\
& Activity pacing 1 (revealing the relation \\
& between pain and behavior) \\
& Activity pacing 2 (activity adjustment by \\
4 & limiting activities and using rest breaks) \\
& Activity pacing 3 (activity adjustment by \\
5 & coping with obstacles) \\
& Cognitive reconstruction 1 (identifying \\
6 & irrational beliefs related to activity \\
& adjusted in sessions 3-5, and distancing) \\
& Cognitive reconstruction 2 (challenging \\
& irrational beliefs) \\
& Summary and relapse prevention
\end{tabular}

CBT-CP Cognitive behavioral therapy for chronic pain, CBT Cognitive behavioral therapy

closely monitored by weekly group supervision using audiotaped recordings of all the sessions conducted in the study.

\section{Statistical analysis}

The data were analyzed following the intent-to-treat principle. To examine the effect of CBT-CP on the primary outcome (EQ5D-5 L), a linear mixed model (LMM) was used. The LMM included a fixed effect of time and a random effect of participants. Time was treated as a categorical variable ( 0 , baseline; 1 , posttreatment; and 2, follow-up). The fixed effect of the baseline primary outcome score was also included in the LMM as covariate. The within-group standardized mean difference (Hedges' $g$ ) and its $90 \%$ confidence interval were calculated using estimated marginal mean and standard error from the LMM. Secondary outcomes were analyzed using the same procedure as used for the primary outcome.

Exploratory analysis was performed that excluded participants with a primary outcome score (EQ5D-5 L) > 0.80 at baseline assessment. This analysis was conducted to estimate the effect size for populations with low QOL. No imputation for missing data was applied because the LMM can provide estimates using all the available data.

Given the preliminary and small size nature of this study, statistical significance was set at 0.10. All analyses were performed using the statistical software $\mathrm{R}$ version 3.5.0 and its packages [52]. LMM was performed using the lmerTest package [53], and standardized mean difference was calculated with the compute.es package [54]. 


\section{Results}

\section{Participant flow, recruitment rate, and dropout rate}

Participants were recruited from 01 November 2016 and the last follow-up assessment was conducted on 23 January 2018. Fig. 1 depicts the participant flow. Of the 24 outpatients, 15 were eligible and were enrolled. When the number of enrollments reached the 15 participants previously targeted, we ended the recruitment. One of them dropped out because of disappearance of pain before starting session 1 . Of the remaining 14, two (14\%) dropped out during the intervention period. The reasons for dropout were family circumstances, after session 3, and seeking other treatment to reduce pain, after session 4. After completing all sessions of CBT-CP, one participant dropped out before follow-up assessment because of the pain getting worse and visiting another hospital. A physician in-charge from another hospital and another physician-in-attendance at the CBT-CP implementation site shared information, and they judged that the above case of exacerbation was not a side effect of CBT-CP. Age, sex, and the scores of primary outcomes (EQ5D-5 L) of the three dropout participants varied (two female and one male; ages, 56, 31, and 61 years; EQ5D-5 L scores, 0.71, 0.67, and 0.80).

\section{Baseline data}

Table 2 presents demographic and clinical characteristics of the participants. Median pain duration was 31 (range, 6-240) months. The anatomical regions affected were the lumbar region (60\%), head (47\%), shoulder (40\%), lower limbs (33\%), and neck (33\%). The number of main diagnoses described in the medical records was 12 , indicating that the chronic pain types targeted in this study were mixed.

\section{Outcome measures}

Table 3 presents the mean and standard deviation of the primary and secondary outcome measures at baseline, post-treatment, and follow-up assessment. Estimated mean differences $(M D)$ and standardized mean differences (Hedges' $g$ ) of the outcome measures with $90 \%$ confidence interval are shown in Table 4.

\section{Primary outcome measures}

The primary outcome (EQ5D-5 L) improved significantly from baseline to post-treatment $(M D=0.10[0.03,0.18])$ and follow-up $(M D=0.09[0.01,0.16])$. The effect size of the EQ5D-5 L was medium from baseline to posttreatment $(g=-0.72[-1.38,-0.05])$ and follow-up $(g=-$ $0.60[-1.22,0.02])$. The confidence intervals were wide.

\section{Secondary outcome measures}

Regarding secondary QOL measures, significant improvement was observed in the SF-12-MCS and SF-12RCS from baseline to post-treatment $(g=-0.80[-1.46$, $-0.13] ; g=-0.94[-1.62,-0.26])$. Between baseline and follow-up, improvement in the SF-12-RCS was statistically significant $(g=-0.80[-1.43,-0.17])$, but that of the SF-12-MCS was not $(g=-0.55[-1.17,0.06])$. The change in the SF-12-PCS was not significant from baseline to post-treatment $(g=-0.18[-0.82,0.47])$ or follow-up $(g=-0.53[-1.15,0.08])$.

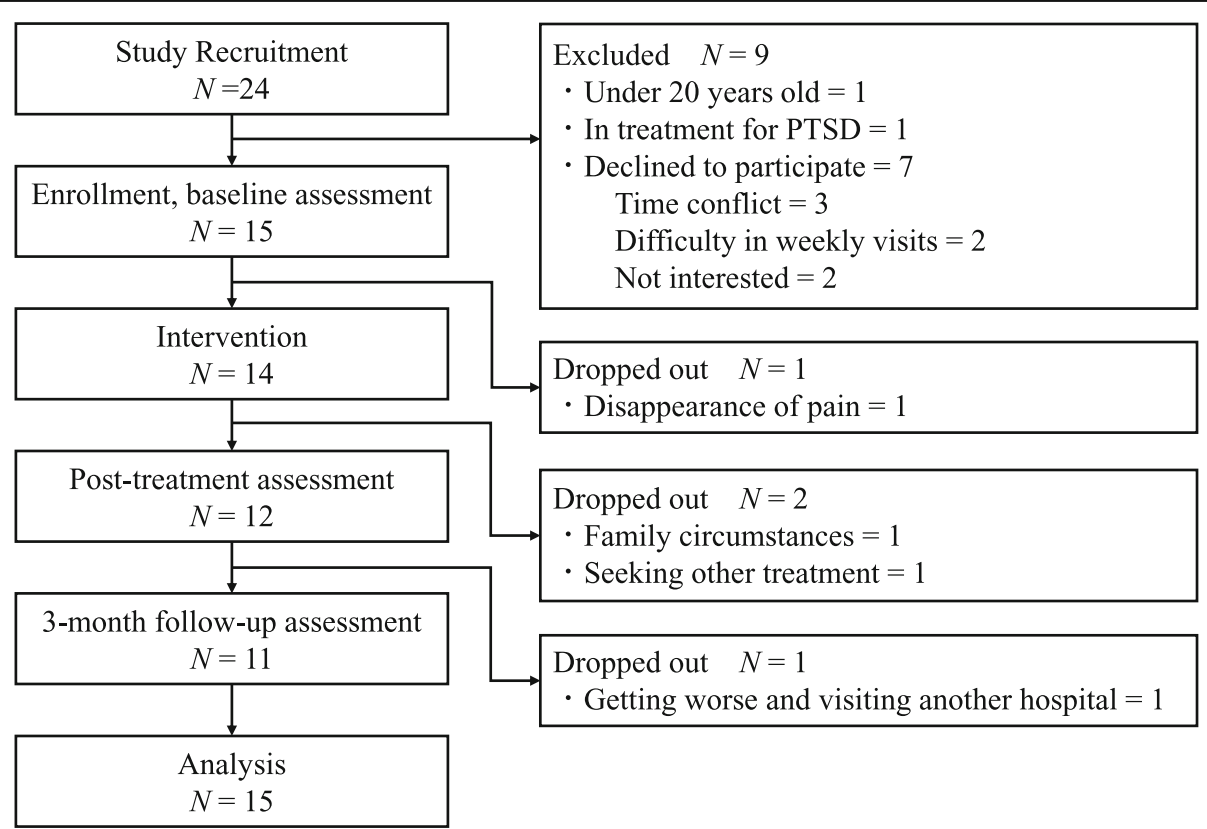

Fig. 1 Participant flow diagram 
Table 2 Participant demographic and clinical characteristics

\begin{tabular}{llll}
\hline & Number & $(\%)$ & [Range] \\
\hline Demographics & 15 & $(100)$ & \\
Age, Mean (SD) & 52.13 & $(14.70)$ & {$[29,76]$} \\
Sex & & & \\
$\quad$ Women & 9 & $(60)$ \\
$\quad$ Men & 6 & $(40)$
\end{tabular}

Education

High-school or less 7

Two year or career college graduate 3

University graduate 4 (27)

Master's degree $\quad 1 \quad$ (7)

Family

Single $\quad 3 \quad$ (20)

With partner 6

With partner and child 2

With Parent $3 \quad$ (20)

With partner, child, and parent $\quad 1 \quad$ (7) Job

$\begin{array}{lll}\text { Full-time worker } & 3 & \text { (20) } \\ \text { Part-time worker } & 1 & (7) \\ \text { Homemaker } & 2 & (13) \\ \text { No job (Older age) } & 2 & (13) \\ \text { No job (Cause of pain) } & 4 & (27) \\ \text { No job (Cause other than pain) } & 2 & \text { (13) } \\ \text { Other } & 1 & \text { (7) }\end{array}$

Pain-related characteristics

Duration (Month), Median

Region of pain (multiple answers allowed)

Lumbar
Head/Face/Mouth
Shoulder/Upper limbs
Lower limbs
Neck
Abdomen
Chest
Genital/Anus/Perineum
Pelvis

Main diagnosis described in a medical record

Chronic primary pain
Fibromyalgia
Adhesive capsulitis
Annal pain
Atypical facial neuralgia
Atypical odontalgia
Cubital tunnel syndrome
Glossodynia
Intercostal neuralgia

Intercostal neuralgia
Table 2 Participant demographic and clinical characteristics (Continued)

\begin{tabular}{llll}
\hline & Number & $(\%)$ & [Range] \\
\hline Occipital neuralgia & 1 & $(7)$ \\
$\begin{array}{l}\text { Ossification of posterior longitudinal } \\
\text { ligament }\end{array}$ & 1 & $(7)$ \\
Sciatica neuralgia & 1 & $(7)$ \\
\hline
\end{tabular}

Regarding pain severity and disability, the NRS summary score and PDAS did not improve significantly from baseline to post-treatment $(g=0.19[-0.45,0.84] ; g=$ $0.51[-0.14,1.16])$ or follow-up $(g=-0.17[-0.78,0.44]$, $g=0.24[-0.36,0.85])$.

Regarding pain-related psychological variables, PCS and PSEQ improved significantly from baseline to posttreatment $(g=0.98[0.30,1.67] ; g=-0.85[-1.52,-$ $0.18])$ and follow-up $(g=1.08$ [0.43, 1.73], $g=-1.03$ [$1.68,-0.39])$. The TSK-11 did not improve significantly from baseline to post-treatment $(g=0.51[-0.14,1.16])$ but showed improvement from baseline to follow-up $(g=0.70[0.07,1.32])$.

Regarding depressive symptoms, the PHQ-9 improved significantly from baseline to post-treatment $(g=0.86$ $[0.19,1.54])$ and follow-up $(g=0.97[0.33,1.62])$.

\section{Adverse events}

No severe adverse events related to study participation occurred during the intervention period, but adverse events were experienced by two participants (family conflict by one and onset of physical illness (endometriosis) by the other). These adverse events were rated as having a non-causal relationship with study participation.

\section{Exploratory analysis}

Table 5 presents estimated and standardized mean differences of the primary and secondary outcomes among 12 participants whose EQ5D-5L scores were $\leq 0.80$. EQ5D-5 L improved significantly from baseline to posttreatment $(\mathrm{MD}=0.18[0.09,0.27])$ and follow-up $(\mathrm{MD}=$ $0.15[0.06,0.24])$. The effect size of the EQ5D-5 L was larger than the entire sample from baseline to posttreatment $(g=-1.24[-2.14,-0.34])$ and follow-up $(g=$ $-1.01[-1.88,-0.14])$. A similar trend was observed in the secondary outcomes.

\section{Discussion}

This pilot study suggests that CBT-CP is feasible for the treatment of chronic pain patients in Japan. CBT-CP promoted statistically significant changes in terms of the primary outcome and some secondary outcomes, with medium-to-large effect sizes. The dropout rate was acceptably low, and no severe adverse events occurred. Most results were in agreement with the hypothesis and indicated that CBT-CP is feasible for use in the Japanese population. 
Table 3 Descriptive statistics of outcome measures (mean and standardized deviation)

\begin{tabular}{|c|c|c|c|c|c|c|}
\hline & \multicolumn{2}{|c|}{ Baseline $(N=15)$} & \multicolumn{2}{|c|}{ Post-treatment $(N=12)$} & \multicolumn{2}{|c|}{ Follow-up $(N=11)$} \\
\hline & Mean & SD & Mean & SD & Mean & SD \\
\hline \multicolumn{7}{|l|}{ Over all QOL measures } \\
\hline EQ5D-5 L (primary outcome) & 0.66 & 0.15 & 0.75 & 0.18 & 0.75 & 0.15 \\
\hline SF-12-PCS & 32.20 & 15.97 & 32.64 & 14.56 & 37.96 & 14.18 \\
\hline SF-12-MCS & 44.89 & 10.70 & 53.00 & 9.74 & 49.96 & 9.39 \\
\hline SF-12-RCS & 39.61 & 12.94 & 47.60 & 13.12 & 47.49 & 13.20 \\
\hline \multicolumn{7}{|l|}{ Pain severity/Disability } \\
\hline NRS summary score & 3.70 & 1.23 & 3.65 & 1.09 & 4.03 & 0.86 \\
\hline PDAS & 18.47 & 10.82 & 15.25 & 9.73 & 16.73 & 14.39 \\
\hline \multicolumn{7}{|l|}{ Pain-related psychological variables } \\
\hline PCS & 30.53 & 11.58 & 19.25 & 11.29 & 17.64 & 10.28 \\
\hline TSK-11 & 26.00 & 5.55 & 23.42 & 4.85 & 22.36 & 4.78 \\
\hline PSEQ & 28.87 & 12.34 & 38.33 & 12.96 & 41.18 & 8.80 \\
\hline \multicolumn{7}{|l|}{ Depressive symptom } \\
\hline PHQ-9 total & 8.73 & 6.09 & 5.25 & 3.91 & 4.64 & 4.30 \\
\hline
\end{tabular}

QOL Quality of life, EQ5D-5 L EuroQol five-dimensional questionnaire five level, SF-12-PCS Medical Outcomes Study 12-Item Short Form Health Survey-Physical Component Summary, MCS Mental Component Summary, RCS Role/Social Component Summary, NRS Numerical Rating Scale, PDAS Pain Disability Assessment Scale, PCS Pain Catastrophizing Scale, TSK-11 Tampa Scale for Kinesiophobia eleven, PSEQ Pain Self-Efficacy Questionnaire, PHQ-9 Patient Health Questionnaire-9

\section{Potential efficacy in terms of QOL, pain severity,} disability, psychological variables, and depressive symptoms

CBT-CP demonstrated medium-to-large effect sizes in terms of QOL. The effect size of EQ5D-5 L as a primary outcome was medium, both post-treatment and at followup. Regarding secondary outcomes, the effect sizes were large for SF-12-MCS post-treatment and for SF-12-RCS at both post-treatment and follow-up. Therefore, mental and role/social health-related QOL improved significantly, and in general, health-related QOL improved with a greater than medium effect size, suggesting that CBT-CP has potential efficacy among Japanese chronic pain patients in terms of QOL improvement. Further, exploratory analysis

Table 4 Estimated mean difference and standardized mean difference with 90\% confidence interval from LMM

\begin{tabular}{|c|c|c|c|c|}
\hline & \multicolumn{2}{|l|}{ Baseline to post-treatment } & \multicolumn{2}{|l|}{ Baseline to follow-up } \\
\hline & MD & SMD (Hedge's g) & MD & SMD(Hedge's g) \\
\hline \multicolumn{5}{|l|}{ Overall QOL measures } \\
\hline EQ5D-5 L (primary outcome) & $0.10[0.03,0.18]$ & $-0.72[-1.38,-0.05]$ & $0.09[0.01,0.16]$ & $-0.60[-1.22,0.02]$ \\
\hline SF-12-PCS & $1.78[-4.06,7.63]$ & $-0.18[-0.82,0.47]$ & $5.49[-0.50,11.48]$ & $-0.53[-1.15,0.08]$ \\
\hline SF-12-MCS & $7.70[2.12,13.28]$ & $-0.80[-1.46,-0.13]$ & $5.45[-0.27,11.17]$ & $-0.55[-1.17,0.06]$ \\
\hline SF-12-RCS & $8.21[3.56,12.87]$ & $-0.94[-1.62,-0.26]$ & $7.10[2.32,11.89]$ & $-0.80[-1.43,-0.17]$ \\
\hline \multicolumn{5}{|l|}{ Pain severity / Disability } \\
\hline NRS summary score & $-0.21[-0.83,0.40]$ & $0.19[-0.45,0.84]$ & $0.19[-0.44,0.82]$ & $-0.17[-0.78,0.44]$ \\
\hline PDAS & $-4.85[-10.33,0.63]$ & $0.51[-0.14,1.16]$ & $-2.34[-7.94,3.26]$ & $0.24[-0.36,0.85]$ \\
\hline \multicolumn{5}{|l|}{ Pain-related psychological variables } \\
\hline PCS & $-11.04[-17.23,-4.85]$ & $0.98[0.30,1.67]$ & $-12.34[-18.69,-5.98]$ & $1.08[0.43,1.73]$ \\
\hline TSK-11 & $-2.83[-6.03,0.37]$ & $0.51[-0.14,1.16]$ & $-3.91[-7.19,-0.63]$ & $0.70[0.07,1.32]$ \\
\hline PSEQ & $9.89[3.66,16.12]$ & $-0.85[-1.52,-0.18]$ & $12.21[5.81,18.61]$ & $-1.03[-1.68,-0.39]$ \\
\hline \multicolumn{5}{|l|}{ Depressive symptoms } \\
\hline PHQ-9 total & $-3.45[-5.75,-1.14]$ & $0.86[0.19,1.54]$ & $-3.95[-6.31,-1.58]$ & $0.97[0.33,1.62]$ \\
\hline
\end{tabular}

LMM Linear mixed model, MD Mean difference, SMD Standardized mean difference (Hedge's g), QOL Quality of life, EQ5D-5L EuroQol five-dimensional questionnaire five level, SF-12-PCS Medical Outcomes Study 12-Item Short Form Health Survey-Physical Component Summary, MCS Mental Component Summary, RCS Role/Social Component Summary, NRS Numerical Rating Scale, PDAS Pain Disability Assessment Scale, PCS Pain Catastrophizing Scale, TSK-11 Tampa Scale for Kinesiophobia eleven, PSEQ Pain Self-Efficacy Questionnaire, PHQ-9 Patient Health Questionnaire-9 
Table 5 Estimated mean difference and standardized mean difference in low QOL participants (EQ5D-5 L $\leq 0.80$ )

\begin{tabular}{|c|c|c|c|c|}
\hline & \multicolumn{2}{|l|}{ Baseline to post-treatment } & \multicolumn{2}{|l|}{ Baseline to follow-up } \\
\hline & $\overline{M D}$ & SMD (Hedge's g) & $\overline{M D}$ & SMD(Hedge's g) \\
\hline \multicolumn{5}{|l|}{ Overall QOL measures } \\
\hline EQ5D-5 L (primary outcome) & $0.18[0.09,0.27]$ & $-1.24[-2.14,-0.34]$ & $0.15[0.06,0.24]$ & $-1.01[-1.88,-0.14]$ \\
\hline SF-12-PCS & $3.97[-4.62,12.56]$ & $-0.32[-1.14,0.50]$ & $7.51[-1.35,16.36]$ & $-0.59[-1.42,0.25]$ \\
\hline SF-12-MCS & $11.01[4.20,17.83]$ & $-1.12[-2.00,-0.23]$ & $7.84[0.74,14.95]$ & $-0.76[-1.61,0.08]$ \\
\hline SF-12-RCS & $12.11[6.21,18.02]$ & $-1.33[-2.24,-0.42]$ & $8.85[2.71,14.98]$ & $-0.94[-1.80,-0.07]$ \\
\hline \multicolumn{5}{|l|}{ Pain severity / Disability } \\
\hline NRS summary score & $-1.00[-1.66,-0.34]$ & $0.96[0.09,1.83]$ & $-0.48[-1.17,0.20]$ & $0.45[-0.38,1.28]$ \\
\hline PDAS & $-8.00[-15.86,-0.14]$ & $0.71[-0.14,1.55]$ & $-3.75[-11.83,4.33]$ & $0.32[-0.50,1.14]$ \\
\hline \multicolumn{5}{|l|}{ Pain-related psychological variables } \\
\hline PCS & $-15.99[-23.54,-8.43]$ & $1.40[0.48,2.32]$ & $-15.76[-23.6,-7.93]$ & $1.34[0.43,2.25]$ \\
\hline TSK-11 & $-3.81[-8.37,0.75]$ & $0.58[-0.26,1.41]$ & $-4.23[-8.96,0.50]$ & $0.62[-0.22,1.46]$ \\
\hline PSEQ & $14.14[5.90,22.38]$ & $-1.12[-2.00,-0.24]$ & $16.03[7.48,24.57]$ & $-1.23[-2.12,-0.33]$ \\
\hline \multicolumn{5}{|l|}{ Depressive symptoms } \\
\hline PHQ-9 total & $-5.56[-8.46,-2.67]$ & $1.33[0.42,2.24]$ & $-4.82[-7.82,-1.83]$ & $1.11[0.23,1.99]$ \\
\hline
\end{tabular}

revealed that the effect size was larger among patients whose QOL was $\leq 0.80$, both post-treatment and at followup. This suggests that patients with low QOL receive more benefit from CBT-CP, as also seen in terms of disability [26].

Although the effect of CBT-CP on pain per se was not assessed, it had a small effect on disability in daily life caused by pain. As shown in a systematic review [15], the effect size for NRS was insignificant and small, both post-treatment and at follow-up. On the other hand, although not significant, the effect size was medium for PDAS post-treatment and for SF-12-PCS at follow-up. A systematic review showed that CBT has a small effect on disability, both in the short and long term [15]. This suggests that CBT-CP is not effective against pain per se, but is potentially effective against disability caused by pain in the Japanese population as well.

CBT-CP contributed significantly to improvement in psychological variables and depressive symptoms related to pain. In a systematic review, CBT has been shown to have a medium effect on catastrophizing in the short term and a small effect in the long term [15]. In this study, CBT-CP had a large effect size on catastrophizing, a medium effect on fear of movement, and a large effect on self-efficacy in managing pain. CBT-CP did not reduce pain per se, but decreased catastrophizing and fear of movement due to pain, thereby empowering patients to manage pain themselves. CBT has been shown to have a small-to-medium effect, both in the short term and the long term, in a systematic review [15]; consistent with this, depressive symptoms were significantly improved both post-treatment and at follow-up in this study. In light of the fear-avoidance model [55], CBT-CP improved catastrophizing, fear of movement, and inactivity, which might reduce depressive symptoms. Thus, CBT may be potentially effective among Japanese chronic pain patients in terms of modifying cognition and attitude toward pain and improving the emotional state.

\section{Potential acceptability and safety of CBT-CP}

The dropout rate was $14 \%$, and there were no severe events that occurred as side effects of CBT-CP during the intervention period. This suggests that the CBT-CP program developed in this study has sufficient acceptability and safety.

\section{Limitations}

There are two limitations of this study. First, it was a before-after trial without a control group. Regarding effect size, although some effect sizes shown in the study were larger than those shown in a systematic review based on RCTs [15], the effect sizes in before-after trials tend to be larger than those of RCTs. Regarding the durability of effect, although there were $>50 \%$ of the variables with medium-to-large effect sizes over a 3-month follow-up, we do not have sufficient data to address the causes. Therefore, future definitive RCTs need to be conducted that address the extent and duration of efficacy of CBT$\mathrm{CP}$ among Japanese chronic pain patients. 
Second, the chronic pain types targeted in this study were mixed. Globally, it is important to examine the effect of CBT-CP on each diagnosis or disease condition constituting chronic pain because there is sufficient evidence that CBT-CP is effective in treating chronic mixed pain [15]. For example, some studies have indicated CBT-CP is effective for chronic low back pain $[12,56,57]$, but not for chronic neck pain [58]. Although differences in cultural background may also have influenced the characteristics of chronic pain and the effect of CBT-CP, studies examining this aspect are insufficient, especially in Asia, including Japan [23]. Therefore, it will be important to conduct more studies on individual chronic pain in Asia following adequate studies on chronic mixed pain.

\section{Conclusions}

CBT is potentially effective for improving QOL and various psychological variables among Japanese chronic pain patients, and the CBT-CP program developed for this study is feasible for use among the Japanese because of low the dropout rate and no severe adverse events related to study participation. Further, development of structured and manualized CBT-CP programs with workbooks and worksheets will contribute not only to future studies but also to clinical practice in Japan. Future definitive RCTs with a control group are needed to carefully examine the effect of CBT among Japanese chronic pain patients, followed by clinical trials addressing each diagnosis and disease condition constituting chronic pain in Asia, including Japan.

\section{Abbreviations}

CBT: Cognitive behavioral therapy; CBT-CP: CBT for chronic pain; QOL: Quality of life; RCT: Randomized controlled trials; UMIN-CTR: University hospital medical information network clinical trials registry; EQ5D-5 L: EuroQol fivedimensional questionnaire five level; SF-12: Medical outcomes in the 12-item short form health survey.; SF-12-PCS: SF-12 physical component summary:; SF-12-MCS: SF-12 mental component summary.; SF-12-RCS: SF-12 role/social component summary.; NRS: Numerical rating scale; PDAS: Pain disability assessment scale; PHQ-9: Patient health questionnaire-9; PCS: Pain catastrophizing scale; TSK-11: Tampa scale for kinesiophobia eleven; PSEQ: Pain self-efficacy questionnaire; LMM: Linear mixed model; MD: Mean differences

\section{Acknowledgments}

The authors would like to thank Noriyo Takahashi for her assistance with the development and trial of versions 1 and 2 of CBT-CP and Enago (www. enago.jp) for the English language review.

\footnotetext{
Authors' contributions

Coordinated the study: HH MS. Conceived and designed the experiments: HH KI TF Y. Takagishi Y. Takebayashi TA YO Y. Tairako MH MS. Reviewed clinical trials and developed the first CBT-CP program: HH KI TF MH. Tried and revised the CBT-CP program: HH KI TF Y. Takagishi TA YO Y. Tairako KE HI IS MH. Recruited the participants as an attending physician: Y. Takao HN AK MK MS. Conducted the CBT-CP program: HH KI TF Y. Takagishi TA Y. Tairako. Supervised: $\mathrm{MH}$. Constructed and managed electronic data capture: KE HI. Analyzed the data: Y. Takebayashi. Drafted the first manuscript: HH Y. Takebayashi (a part of methods and results). Examined and critically contributed to and finally approved the manuscript: HH KI TF Y. Takagishi Y. Takebayashi TA YO Y. Tairako Y. Takao HN AK MK KE HI IS MH MS.
}

\section{Funding}

This study was supported by the Japan Agency for Medical Research and Development (AMED) under Grant Number JP16ek0610004. The funder of this study had no role in the study design, data collection, data analysis, data interpretation, or writing of this manuscript.

\section{Availability of data and materials}

The datasets used and/or analyzed during the current study are available from the corresponding author on reasonable request.

\section{Ethics approval and consent to participate}

Ethics committees at Osaka University Hospital, Kobe University Hospital, Kawasaki Medical School Hospital, National Center of Neurology and Psychiatry Hospital, and Jikei University Hospital evaluated the protocols (approval numbers 15429-2; 280046; 2275-2; A2016-092; and 28-256, respectively). All participants provided written informed consent.

\section{Consent for publication}

Not applicable.

\section{Competing interests}

The authors declare that they have no competing interests.

\section{Author details}

${ }^{1}$ Department of Sociology, Faculty of Sociology, Kansai University, 3-3-35 Yamate-cho, Suita-shi, Osaka 564-8680, Japan. ${ }^{2}$ National Center for Cognitive Behavior Therapy and Research, National Center of Neurology and Psychiatry, 4-1-1 Ogawahigashi-cho, Kodaira-shi, Tokyo 187-8551, Japan. ${ }^{3}$ Faculty of Education, Shujitsu University, 1-6-1 Nishigawara, Naka-ku, Okayama-shi, Okayama 703-8516, Japan. ${ }^{4}$ Graduate School of Integrated Arts and Sciences, Tokushima University, 1-1 Minamijosanjima-cho, Tokushima-shi, Tokushima 770-8502, Japan. ${ }^{5}$ Department of Psychology, Surugadai University, 698 Azu, Hanno-shi, Saitama 357-8555, Japan. ${ }^{6}$ Department of Health Risk

Communication, School of Medicine, Fukushima Medical University, 1 Hikarigaoka, Fukushima-shi, Fukushima 960-1295, Japan. ${ }^{7}$ Pain Management Clinic, Shiga University of Medical Science Hospital, Seta Tsukinowa-cho, Otsu-shi, Shiga 520-2192, Japan. ${ }^{8}$ Department of Neuropsychiatry, Kyorin University School of Medicine, 6-20-2 Shinkawa, Mitaka-shi, Tokyo 181-8611, Japan. ${ }^{9}$ Department of Psychology, Meiji Gakuin University, 1-2-37 Shirokanedai, Minato-ku, Tokyo 108-8636, Japan. ${ }^{10}$ Department of Pain Medicine, Hyogo College of Medicine, 1-1 Mukogawa-cho, Nishinomiya-shi, Hyogo 663-8501, Japan. ${ }^{11}$ Anesthesiology and Intensive Care 2, Kawasaki Medical School, 577 Matsushima, Kurashiki-shi, Okayama 701-0192, Japan. ${ }^{12}$ Department of Anesthesiology, Yokohama City University Medical Center, 4-57 Urafune-cho, Minami-ku, Yokohama-shi, Kanagawa 232-0024, Japan.

${ }^{13}$ Graduate School of Human Sciences, Osaka University, 1-2 Yamadaoka, Suita-shi, Osaka 565-0871, Japan. ${ }^{14}$ Counseling Office, Japan Women's University, 1-1-1 Nishiikuta, Tama-ku, Kawasaki-shi, Kanagawa 214-8565, Japan. ${ }^{15}$ Department of Neurology, National Center of Neurology and Psychiatry, 4-1-1 Ogawahigashi-cho, Kodaira-shi, Tokyo 187-8553, Japan. ${ }^{16}$ TCBT Counseling Office, Cosmos Kichijoji Building 2F, 2-8-4 Kichijojihoncho, Musashino-shi, Tokyo 180-0004, Japan. ${ }^{17}$ Department of Health Science, Naragakuen University, 3-15-1 Nakatomigaoka, Nara-shi, Nara 631-8524, Japan.

Received: 5 September 2019 Accepted: 28 February 2020

Published online: 10 March 2020

References

1. Merskey H, Bogduk N. Classification of chronic pain. 2nd ed. Seattle: IASP Press; 1994.

2. Treede RD, Rief W, Barke A, Aziz Q, Bennett MI, Benoliel R, Cohen M, Evers S, Finnerup NB, First MB, et al. A classification of chronic pain for ICD-11. Pain. 2015;156(6):1003-7.

3. Inoue S, Kobayashi F, Nishihara M, Arai YC, Ikemoto T, Kawai T, Inoue M, Hasegawa T, Ushida T. Chronic pain in the Japanese community - prevalence, characteristics and impact on quality of life. PLoS One. 2015:10(6):e0129262.

4. Vlaeyen JW, Morley S, Crombez G. The experimental analysis of the interruptive, interfering, and identity-distorting effects of chronic pain Behav Res Ther. 2016;86:23-34. 
5. Goldberg DS, McGee SJ. Pain as a global public health priority. BMC Public Health. 2011;11:770.

6. Institute of Medicine. Relieving pain in America: a blueprint for transforming prevention, care, education, and research. Washington, DC: The National Academies Press; 2011.

7. Gaskin DJ, Richard P. The economic costs of pain in the United States. J Pain. 2012;13(8):715-24.

8. Scascighini L, Toma V, Dober-Spielmann S, Sprott H. Multidisciplinary treatment for chronic pain: a systematic review of interventions and outcomes. Rheumatology. 2008;47(5):670-8.

9. Kerns RD, Sellinger J, Goodin BR. Psychological treatment of chronic pain. Annu Rev Clin Psychol. 2011;7:411-34.

10. Turner JA, Romano JM. Cognitive-behavioral therapy for chronic pain. In: Loeser JD, Bonica JJ, editors. Bonica's management of pain. 3rd ed. Philadelphia, PA: Lippincott Williams \& Wilkins; 2001. p. 1751-8.

11. Turner JA, Mancl L, Aaron LA. Short- and long-term efficacy of brief cognitive-behavioral therapy for patients with chronic temporomandibular disorder pain: a randomized, controlled trial. Pain. 2006;121(3):181-94.

12. Linden M, Scherbe S, Cicholas B. Randomized controlled trial on the effectiveness of cognitive behavior group therapy in chronic back pain patients. J Back Musculoskelet Rehabil. 2014;27(4):563-8.

13. Buhrman M, Syk M, Burvall O, Hartig T, Gordh T, Andersson G. Individualized guided internet-delivered cognitive-behavior therapy for chronic pain patients with comorbid depression and anxiety: a randomized controlled trial. Clin J Pain. 2015;31(6):504-16.

14. Cherkin DC, Sherman KJ, Balderson BH, Cook AJ, Anderson ML, Hawkes RJ, Hansen KE, Turner JA. Effect of mindfulness-based stress reduction vs cognitive behavioral therapy or usual care on back pain and functional limitations in adults with chronic low back pain: a randomized clinical trial. JAMA. 2016;315(12):1240-9.

15. Williams AC, Eccleston C, Morley S. Psychological therapies for the management of chronic pain (excluding headache) in adults. Cochrane Database Syst Rev. 2012;11:CD007407.

16. Castro MM, Daltro C, Kraychete DC, Lopes J. The cognitive behavioral therapy causes an improvement in quality of life in patients with chronic musculoskeletal pain. Arq Neuropsiquiatr. 2012;70(11):864-8.

17. Matsudaira K, Takeshita K, Kunoki J, Yamazaki T, Hara N, Yamada K, Takagi Y. Prevalence and characteristics of chronic pain in the general Japanese population (in Japanese). Pain Clinic. 2011;32(9):1345-56.

18. Commitee for Clinical Practice Guideline for Chronic Pain. Clinical practice guideline for chronic pain. Tokyo: Shinko Trading Co. Ltd; 2018.

19. Japanese Orthopaedic Association, Japanese Society of Lumbar Spine Disorders. Clinical practice guideline for lumbar spine disorders [translated from Japanese]. Tokyo: Nankodo; 2012.

20. Japan College of Fibromyalgia Investigation. Clinical practice guideline for fybromyalgia 2013 [translated from Japanese]. Tokyo: Japan Medical Journal; 2013.

21. Nakamura M, Nishiwaki Y, Ushida T, Toyama Y. Prevalence and characteristics of chronic musculoskeletal pain in Japan. J Orthop Sci. 2011; 16(4):424-32.

22. Matsubara T, Arai YC, Shimo K, Sato J, Osuga T, Nishihara M, Ushida T. Effects of cognitive-behavioral therapy on pain intensity and level of physical activity in Japanese patients with chronic pain- a preliminary quasiexperimental study. J Phys Ther. 2010;1(2):49-57.

23. Yang SY, McCracken LM, Moss-Morris R. Psychological treatments for chronic pain in east and Southeast Asia: a systematic review. Int J Behav Med. 2016:23(4):473-84.

24. Yoshino A, Okamoto Y, Doi M, Horikoshi M, Oshita K, Nakamura R, Otsuru N, Yoshimura S, Tanaka K, Takagaki K, et al. Effectiveness of group cognitive behavioral therapy for somatoform pain disorder patients in Japan: a preliminary non-case-control study. Psychiatry Clin Neurosci. 2015;69(12): 763-72.

25. Otto MW, Smits JA, Reese HE. Cognitive-behavioral therapy for the treatment of anxiety disorders. J Clin Psychiatry. 2004;65(Suppl 5):34-41.

26. Hill JC, Whitehurst DG, Lewis M, Bryan S, Dunn KM, Foster NE, Konstantinou K, Main CJ, Mason E, Somerville S, et al. Comparison of stratified primary care management for low Back pain with current best practice (STarT Back): a randomised controlled trial. Lancet. 2011:378(9802):1560-71.

27. EuroQol Group. EuroQol-a new facility for the measurement of healthrelated quality of life. Health policy. 1990;16(3):199-208.
28. Shiroiwa T, Ikeda S, Noto S, Igarashi A, Fukuda T, Saito S, Shimozuma K. Comparison of value set based on DCE and/or TTO data: scoring for EQ-5D$5 \mathrm{~L}$ health states in Japan. Value Health. 2016;19(5):648-54.

29. Ware JEJ, Kosinski M, Keller SD. A 12-item short-form health survey: construction of scales and preliminary tests of reliability and validity. Med Care. 1996;34(3):220-33.

30. Suzukamo Y, Fukuhara S, Green J, Kosinski M, Gandek B, Ware JE. Validation testing of a three-component model of short Form-36 scores. J Clin Epidemiol. 2011;64(3):301-8.

31. Jensen MP, Turner JA, Romano JM, Fisher LD. Comparative reliability and validity of chronic pain intensity measures. Pain. 1999;83(2):157-62.

32. Serlin RC, Mendoza TR, Nakamura Y, Edwards KR, Cleeland CS. When is cancer pain mild, moderate or severe? Grading pain severity by its interference with function. Pain. 1995;61(2):277-84.

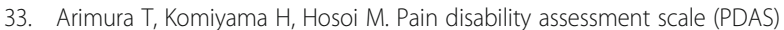
- a simplified scale for clinical use — (in Japanese). Japanese J Behav Ther. 1997;23(1):7-15.

34. Yamashiro K, Arimura T, Iwaki R, Jensen MP, Kubo C, Hosoi M. A multidimensional measure of pain interference: reliability and validity of the pain disability assessment scale. Clin J Pain. 2010;27(4):338-43.

35. Spitzer RL, Kroenke K, Williams JB. Validation and utility of a self-report version of PRIME-MD: the PHQ primary care study. JAMA. 1999;282(18): 1737-44.

36. Muramatsu K, Miyaoka H, Kamijima K, Muramatsu Y, Yoshida M, Otsubo T, Gejyo F. The patient health questionnaire, Japanese version: validity according to the mini-international neuropsychiatric interview-plus. Psychol Rep. 2007;101:952-60.

37. Sullivan MJL, Bishop SR, Pivik J. The pain Catastrophizing scale: development and validation. Psychol Assess. 1995;7(4):524-32.

38. Osman A, Barrios FX, Gutierrez PM, Kopper BA, Merrifield T, Grittmann L. The pain Catastrophizing scale: further psychometric evaluation with adult samples. J Behav Med. 2000;23(4):351-65.

39. Matsuoka H, Sakano Y. Assessment of cognitive aspect of pain: development, reliability, and validation of Japanese version of pain catastrophizing scale (in Japanese). Jpn J Psychosom Med. 2007;47:95-102.

40. Kikuchi N, Matsudaira K, Sawada T, Oka H. Psychometric properties of the Japanese version of the Tampa scale for Kinesiophobia (TSK-J) in patients with whiplash neck injury pain and/or low back pain. J Orthop Sci. 2015; 20(6):985-92.

41. Nicholas MK. The pain self-efficacy questionnaire: taking pain into account. Eur J Pain. 2007;11(2):153-63

42. Adachi T, Nakae A, Maruo T, Shi K, Shibata M, Maeda L, Saitoh Y, Sasaki J. Validation of the Japanese version of the pain self-efficacy questionnaire in Japanese patients with chronic pain. Pain Med. 2014;15(8):1405-17.

43. Moore CG, Carter RE, Nietert PJ, Stewart PW. Recommendations for planning pilot studies in clinical and translational research. Cts-Clin Transl Sci. 2011:4(5):332-7.

44. Kerns RD, Burns JW, Shulman M, Jensen MP, Nielson WR, Czlapinski R, Dallas MI, Chatkoff D, Sellinger J, Heapy A, et al. Can we improve cognitivebehavioral therapy for chronic back pain treatment engagement and adherence? A controlled trial of tailored versus standard therapy. Health Psychol. 2013;33(9):938-47.

45. Nicholas MK, Asghari A, Sharpe L, Brnabic A, Wood BM, Overton S, Tonkin L, de Sousa M, Finniss D, Beeston L, et al. Cognitive exposure versus avoidance in patients with chronic pain: adherence matters. Eur J Pain. 2014;18(3):424-37.

46. Kjeldgaard D, Forchhammer HB, Teasdale TW, Jensen RH. Cognitive behavioural treatment for the chronic post-traumatic headache patient: a randomized controlled trial. J Headache Pain. 2014;15:81.

47. Monticone M, Baiardi P, Vanti C, Ferrari S, Nava T, Montironi C, Rocca B, Foti C, Teli M. Chronic neck pain and treatment of cognitive and behavioural factors: results of a randomised controlled clinical trial. Eur Spine J. 2012; 21(8): $1558-66$

48. Tang NK, Goodchild CE, Salkovskis PM. Hybrid cognitive-behaviour therapy for individuals with insomnia and chronic pain: a pilot randomised controlled trial. Behav Res Ther. 2012;50(12):814-21.

49. Buhrman M, Nilsson-Ihrfeldt E, Jannert M, Strom L, Andersson G. Guided internet-based cognitive behavioural treatment for chronic back pain reduces pain catastrophizing: a randomized controlled trial. J Rehabil Med. 2011:43(6):500-5. 
50. Day MA, Thorn BE, Kapoor S. A qualitative analysis of a randomized controlled trial comparing a cognitive-behavioral treatment with education. J Pain. 2011;12(9):941-52.

51. Wetherell JL, Afari N, Rutledge T, Sorrell JT, Stoddard JA, Petkus AJ, Solomon BC, Lehman DH, Liu L, Lang AJ, et al. A randomized, controlled trial of acceptance and commitment therapy and cognitive-behavioral therapy for chronic pain. Pain. 2011;152(9):2098-107.

52. R Core Team. R: a language and environment for statistical computing. Vienna, R Foundation for Statistical Computing; 2018.

53. Kuznetsova A, Brockhoff PB, Christensen RHB. ImerTest package: tests in linear mixed effects models. J Stat Softw. 2017;82(13):1-26.

54. Del Re AC. compute.es: compute effect sizes. R package version 0.2-2. R Package 2013. https://cran.r-project.org/web/packages/compute.es.

55. Lethem J, Slade PD, Troup JD, Bentley G. Outline of a fear-avoidance model of exaggerated pain perception--I. Behav Res Ther. 1983;21(4):401-8.

56. Turner JA, Anderson ML, Balderson BH, Cook AJ, Sherman KJ, Cherkin DC. Mindfulness-based stress reduction and cognitive behavioral therapy for chronic low back pain: similar effects on mindfulness, catastrophizing, selfefficacy, and acceptance in a randomized controlled trial. Pain. 2016;157(11): 2434-44.

57. Richmond H, Hall AM, Copsey B, Hansen Z, Williamson E, Hoxey-Thomas N, Cooper Z, Lamb SE. The effectiveness of cognitive behavioural treatment for non-specific low back pain: a systematic review and meta-analysis. PLoS One. 2015;10(8):e0134192.

58. Monticone M, Ambrosini E, Cedraschi C, Rocca B, Fiorentini R, Restelli M, Gianola S, Ferrante S, Zanoli G, Moja L. Cognitive-behavioral treatment for subacute and chronic neck pain: a cochrane review. Spine. 2015;40(19): 1495-504.

\section{Publisher's Note}

Springer Nature remains neutral with regard to jurisdictional claims in published maps and institutional affiliations.

Ready to submit your research? Choose BMC and benefit from:

- fast, convenient online submission

- thorough peer review by experienced researchers in your field

- rapid publication on acceptance

- support for research data, including large and complex data types

- gold Open Access which fosters wider collaboration and increased citations

- maximum visibility for your research: over $100 \mathrm{M}$ website views per year

At $\mathrm{BMC}$, research is always in progress.

Learn more biomedcentral.com/submissions 